\title{
BAG-I predicts patient outcome and tamoxifen responsiveness in ER-positive invasive ductal carcinoma of the breast
}

\author{
EKA Millar, 1,2, LR Anderson', CM McNeil ${ }^{1,3}$, SA O'Toole ${ }^{1,4}$, M Pinese', P Crea $^{5}$, AL Morey ${ }^{4}$, AV Biankin ${ }^{1,6,7}$, \\ SM Henshall ${ }^{1,6}$, EA Musgrove ${ }^{1,6}$, RL Sutherland ${ }^{1,6}$ and AJ Butt ${ }^{1,6}$
}

'Cancer Research Program, Garvan Institute of Medical Research, Darlinghurst, Sydney, New South Wales 20 I 0, Australia; ${ }^{2}$ Department of Anatomical Pathology, South Eastern Area Laboratory Service, St George Hospital, Kogarah, New South Wales 2217, Australia; ${ }^{3}$ Department of Medical Oncology, Westmead Hospital, University of Sydney, Westmead, New South Wales 21 45, Australia; ${ }^{4}$ Department of Pathology (SydPath), St Vincent's Hospital, Darlinghurst, Sydney, New South Wales 20 10, Australia; ${ }^{5}$ Department of Surgical Oncology, St Vincent's Hospital, Darlinghurst, Sydney, New South Wales 20 10, Australia; ${ }^{6}$ Faculty of Medicine, St Vincent's Clinical School, University of NSW. New South Wales 2052, Australia; ${ }^{7}$ Division of Surgery, Bankstown Hospital, Bankstown, New South Wales 2200, Australia

BAG-I (bcl-2-associated athanogene) enhances oestrogen receptor (ER) function and may influence outcome and response to endocrine therapy in breast cancer. We determined relationships between BAG-I expression, molecular phenotype, response to tamoxifen therapy and outcome in a cohort of breast cancer patients and its influence on tamoxifen sensitivity in MCF-7 breast cancer cells in vitro. Publically available gene expression data sets were analysed to identify relationships between BAG-I mRNA expression and patient outcome. BAG-I protein expression was assessed using immunohistochemistry in 292 patients with invasive ductal carcinoma and correlated with clinicopathological variables, therapeutic response and disease outcome. BAG-I-overexpressing MCF-7 cells were treated with antioestrogens to assess its effects on cell proliferation. Gene expression data demonstrated a consistent association between high BAG-I mRNA and improved survival. In ER + cancer $(n=189)$, a high nuclear BAG-I expression independently predicted improved outcome for local recurrence $(P=0.0464)$, distant metastases $(P=0.0435)$, death from breast cancer $(P=0.009$, hazards ratio $0.29,95 \% \mathrm{Cl}: 0.114-0.735)$ and improved outcome in tamoxifen-treated patients $(n=107 ; P=0.0191)$. BAG-I overexpression in MCF-7 cells augmented antioestrogen-induced growth arrest. A high BAG-I expression predicts improved patient outcome in ER + breast carcinoma. This may reflect both a better definition of the hormoneresponsive phenotype and a concurrent increased sensitivity to tamoxifen.

British Journal of Cancer (2009) 100, 123-133. doi: 10.1038/sj.bjc.6604809 www.bjcancer.com

Published online 9 December 2008

(c) 2009 Cancer Research UK

Keywords: breast cancer; prognosis; response marker; BAG-I; tamoxifen sensitivity

Breast cancer is a heterogeneous disease with considerable variability in clinical outcome, the prognosis and management of which is largely based on histopathological features accompanied by established markers of hormone receptor status, oestrogen and progesterone receptors (oestrogen receptor (ER), progesterone receptor (PR)), and HER-2 amplification (Sorlie et al, 2001; Goldhirsch et al, 2007). Oestrogen receptor-positive disease comprises approximately $70 \%$ of cases and therapies targeting oestrogen synthesis or the ER are the most effective treatments, with adjuvant tamoxifen reducing the annual risk of recurrence and death by up to 47 and 26\% respectively (Early Breast Cancer Trialists' Collaborative Group, 2005) and reducing the risk of contralateral disease by $50 \%$ (Fisher et al, 1998). However, the benefits of treatment are limited by intrinsic or acquired resistance, which occurs in approximately $40 \%$ of ER + breast cancers (Howell et al, 2005). New predictive biomarkers of hormone responsiveness and disease outcome are needed to improve selection of patients for optimal 'targeted' endocrine

*Correspondence: Dr EKA Millar; E-mail: e.millar@garvan.org.au Received 16 September 2008; revised 12 November 2008; accepted 12 November 2008; published online 9 December 2008 therapy at an earlier stage in the disease process with potential survival benefits. In addition, they may also identify key mechanisms involved in antioestrogen resistance/sensitivity.

Gene expression profiling has identified intrinsic molecular phenotypes of breast cancer that subclassify ER + tumours into two main subtypes that predict outcome: luminal A and B, which can be distinguished by the presence of increased proliferation, HER-2 amplification and a less favourable prognosis in the latter group (Sorlie et al, 2001). Signatures that predict outcome in ER + disease treated with tamoxifen (Ma et al, 2004; Jansen et al, 2005; Loi et al, 2008) have been useful in identifying potential new predictive biomarkers. However, such molecular testing is expensive and there is often little overlap between signatures from different studies. Furthermore, translating these findings into clinically useful biomarkers suitable for routine pathology practice is a priority. Ideally, this would be performed using immunohistochemistry, which is more cost-effective and more easily introduced within the existing infrastructure. However, this approach is often limited by the lack of commercially available antibodies for many of these genes.

BAG-1 (bcl-2-associated athanogene) is a pro-survival protein that can influence diverse biological processes including nuclear hormone receptor function, apoptosis, signal transduction and 
protein turnover (reviewed in Cutress et al (2002)). BAG-1 exists as three protein isoforms. The specific ability of the long isoform, BAG-1L ( $\mathrm{p} 50$ ), which possesses a nuclear localisation sequence not present in the other isoforms, to upregulate the transcriptional activity of both ER $\alpha$ and $\mathrm{ER} \beta$ up to five-fold in MCF-7 breast cancer cells (Cutress et al, 2003), is of potential functional and prognostic significance. BAG-1 is expressed in most normal human tissues (Takayama et al, 1998), and its overexpression has been described not only in breast cancer, but also in other human malignancies including squamous cell carcinoma of the head and neck (Shindoh et al, 2000), chronic lymphocytic leukaemia (Kitada et al, 1998) and prostate cancer (Maki et al, 2007), in which it is associated with a poor prognosis. However, its role as a predictive marker in breast cancer has not been established. Several studies have attempted to relate BAG-1 protein expression to disease outcome with inconsistent results, which may have been the result of low patient numbers, low rates of ER + tumours (ER + rates of $35-52 \%$ rather than a currently expected rate of $\sim 70 \%$ ) and incomplete pathological, clinical and treatment information. However, the improved prognosis associated with a high $B A G-1$ expression has earlier been demonstrated in three studies although with differences in subcellular localisation of BAG-1 expression that is, cytoplasmic (Turner et al, 2001), nuclear (Cutress et al, 2003), and cytoplasmic or nuclear (Nadler et al, 2008). More recently, BAG-1 featured as one of the 16 cancerspecific genes included in the Oncotype Dx assay (Paik et al, 2004), which predicts distant failure in $\mathrm{ER}+$, lymph node-negative patients treated with tamoxifen using PCR of formalin-fixed paraffin-embedded (FFPE) material. In addition, this assay has also been used to predict the potential benefit of chemotherapy (Paik et al, 2006) in this group of patients.

As ER-mediated regulation of cell growth, proliferation and survival are key components of breast cancer development, the role of BAG-1 as a predictive and prognostic marker in breast cancer requires further investigation. Consequently, we aimed to define the relationship of BAG-1 expression with outcome and response to therapy in a large cohort of early breast cancer patients of uniform histological type with well-documented treatment and follow-up data.

\section{MATERIALS AND METHODS}

\section{BAG-1 mRNA expression and outcome}

Publically available gene expression data from two published studies (van de Vijver et al, 2002; Naderi et al, 2007) of breast cancer outcome were analysed to initially identify a potential relationship between BAG-1 mRNA levels and prognosis. The cohorts chosen for these analyses were of similar clinicopathological composition to our clinical cohort. The study by Naderi et al (2007) comprised 135 patients, $70 \%$ of whom were ER + with a median follow-up of 132 months (range 16-160 months). Data were generated using Agilent Human 1A arrays, which were available as raw scanner data files and sourced from Array Express (http://www.ebi.ac.uk/) accession E-UCON-1. Using the limma R package ( $\mathrm{R}$ Development Core Team, 2007), background-subtracted data were normalised by the global LOESS technique applied to non-control spots only. To combine information from duplicate dye-swap arrays, a linear model was fitted to the normalised data using limma (Smyth, 2005). Model fit coefficients for each sample were then used as final expression estimates, expressed relative to a pooled reference RNA. The second data set, sourced from van de Vijver et al (2002), comprised 295 patients, $76 \%$ of which were ER +, with a median follow-up of 93.6 months (range 0.6-220 months). Data were generated using Rosetta NKIspotted oligonucleotide arrays and were downloaded from http:// microarray-pubs.stanford.edu/wound_NKI/ explore.html as $\log 2$ transformed values in a text table format. Raw data were directly transferred to the final output file without further processing. One BAG-1 probe set was available from each cohort and expression data were analysed for frequency distribution of mRNA and its association with patient outcome.

\section{Patient characteristics}

BAG-1 protein expression was assessed by immunohistochemistry in tumours from a cohort of 292 patients diagnosed with invasive ductal breast carcinoma and treated by a single surgeon (PC) between February 1992 and August 2002. Formalin-fixed, paraffinembedded tissue was retrieved from St Vincent's Public Hospital (Sydpath) and St Vincent's Private Hospital (Douglas Hanly Moir Pathology), Sydney, Australia. All tumours were classified as invasive ductal carcinoma of no special type and graded using standardised histological criteria (Elston and Ellis, 1991). Lymph node status was assessed by axillary sampling and histological examination. Follow-up intervals were calculated from the date of definitive procedure (biopsy/lumpectomy/mastectomy) to the date of last-recorded follow-up (median 64 months, range 0-152 months). Patients less than 50 years of age with node-positive, ER- tumours or tumours larger than $3 \mathrm{~cm}$ received adjuvant chemotherapy (cyclophosphamide, methotrexate and 5-fluorouracil or adriamycin and cyclophosphamide (AC)). Patients with $\mathrm{ER}+$ tumours who were more than 50 years of age received 5 years of tamoxifen therapy. Breast cancer-specific survival was defined as date of definitive procedure to date of death due to breast cancer. Patients who died of causes unrelated to breast cancer were considered as censored at the time of death. Deaths from unknown causes were excluded from analysis of diseasespecific survival. Recurrences were confirmed by imaging and/or histology. Locoregional recurrences were defined as of the ipsilateral breast, chest wall, axilla or supraclavicular fossa. Distant relapses and metastases were defined as disease in the lungs, liver, brain or distant lymph nodes. These data were obtained from annual review of patient files or cancer registry data. Tissue microarrays (TMAs) of FFPE tumour tissue blocks were constructed with approximately $80 \times 1 \mathrm{~mm}$ cores per slide. Each patient was represented by two to six $1 \mathrm{~mm}$ cores. Prior approval for this study was obtained from the Human Research Ethics Committee of St Vincent's Hospital, Sydney (HREC SVH H94/080, HREC 06336 SVH H00 036).

\section{Immunohistochemistry}

Four-micron sections were cut from each TMA, mounted on SuperFrost ${ }^{\circledR}$ Plus glass slides and baked for $2 \mathrm{~h}$ at $79^{\circ} \mathrm{C}$, then dewaxed by passage through xylene (two $5 \mathrm{~min}$ washes), cleared and rehydrated in graded alcohol (100, 95 and 70\%) ending in a distilled water wash. Antigen retrieval was performed using DAKO solution ( $\mathrm{pH}$ 6.0) (s1699; DAKO, Carpentaria, CA, USA) in a pressure cooker (DAKO Pascal Decloaker) for $60 \mathrm{~s}$, followed by cooling gently for $15 \mathrm{~min}$ in a running water bath. Following a thorough wash in distilled water, endogenous peroxidase activity was eliminated with $3 \%$ hydrogen peroxide for $5 \mathrm{~min}$. Slides were incubated with BAG-1 mouse monoclonal antibody raised against full-length human BAG-1 protein (clone 3.10G3E2; Santa Cruz Biotechnology Inc., Santa Cruz, CA, USA) at a dilution of 1:50 for $45 \mathrm{~min}$ at room temperature. Following buffer wash, detection employed DAKO Envision + mouse secondary reagent (DAKO) for $30 \mathrm{~min}$ at room temperature, followed by DAKO DAB + chromagen (DAKO) for $10 \mathrm{~min}$. Slides were then rinsed in water and counterstained with haematoxylin, dehydrated through graded ethanol, cleared in xylene and mounted. Normal colon was employed as a control tissue that showed positive staining in basal crypt cell nuclei and negative staining in the muscularis mucosae. A further negative control substituted isotype-matched mouse IgG1 at 1:100 in place of the BAG-1 monoclonal antibody. 
Oestrogen receptor, PR, cytokeratin 5/6 and EGFR were also stained using the following antibodies: ER, 1:100 (clone 6F11; DAKO); PR, 1:200 (clone PgR 636; DAKO); CK5/6, 1:80 (clone MAB1602; Chemicon International, Temecula, CA, USA); and EGFR, 1:100 (clone H11; DAKO). HER-2 FISH was assessed in the Australian National Reference Laboratory (Department of Pathology, St Vincent's Hospital, Sydney) using the Vysis PathVysion HER-2 DNA dual-colour probe kit. An HER2: chromosome 17 ratio $>2.2$ was classified as HER2 amplification.

All assessments of immunohistochemical staining were performed by observers blinded to the clinical and molecular data and patient outcome. Nuclear and cytoplasmic staining for BAG-1 was assessed by an experienced breast pathologist (EKAM) and described in terms of the intensity ( 0 : negative, $1+$ : weak, $2+$ : moderate and $3+$ : strong) and percentage of cells staining positive. From these indices, a simplified ' $H$ score' (i.e., intensity $\times$ percentage of positive nuclei) was calculated for each core and a mean and median score for each parameter calculated for each tumour (range of two to six cores per patient). Oestrogen receptor and PR (both double scored) were assessed as positive if they had a simplified $H$ score of $>10$. CK5/6 and EGFR (both double scored) were assessed as positive if there was any positive cytoplasmic or membranous staining present at any intensity.

\section{Definition of intrinsic molecular phenotype of breast cancer}

This was assessed immunohistochemically using criteria similar to those recently described by Cheang et al (2008) but using FISH to determine HER-2 status as follows: luminal $\mathrm{A}=\mathrm{ER}+$ and/or $\mathrm{PR}+$, HER-2-; luminal $\mathrm{B}=\mathrm{ER}+$ and/or $\mathrm{PR}+$, HER-2 + ; HER-2 $=\mathrm{ER}-$ and $\mathrm{PR}-, \mathrm{HER}-2+$; basal-like $=\mathrm{ER}-, \mathrm{PR}-$, HER-2 - , CK5/6 + and/or EGFR + ; unclassified = negative for all five markers.

\section{Cell culture studies}

The human ER + breast cancer cell line, MCF-7, was routinely maintained in RPMI-1640 medium supplemented with 5\% foetal calf serum, $10 \mu \mathrm{g} \mathrm{ml}^{-1}$ insulin and $2.92 \mathrm{mg} \mathrm{m}^{-1}$ glutamine under standard conditions. A cDNA insert encoding human BAG-1 (cat no. SC107955; OriGene Technologies Inc., Rockville, MD, USA) was cloned into the retroviral vector pMSCV-IRES-GFP (Caldon et al, 2008). MCF-7 cells transiently expressing the murine ecotropic receptor were infected with BAG-1 retrovirus as described earlier (Debnath et al, 2003). Green fluorescent protein-positive cells were sorted to homogeneity by flow cytometry. Cell lysates were collected as described earlier (Prall et al, 1997). Subsequent western blotting using a BAG-1 antibody
(3.10G3E2; Clone Chemicon International Inc., Billerica, MA, USA) confirmed BAG-1 expression. $\beta$-Actin (Sigma, St Louis, MO, USA) was used as a loading control.

\section{S-phase analysis}

Exponentially growing MCF-7 cells expressing BAG-1 or vectoralone control were treated with $1 \mu \mathrm{moll}^{-1}$ 4-hydroxytamoxifen (Sigma) or $10 \mathrm{nmoll}^{-1}$ ICI 182780 ( $7 \alpha-[9-(4,4,5,5,5$-pentafluoropentylsulphinyl) nonyl] estra-1,3,5,(10)-triene-3,17 $\beta$-diol), which was a kind gift from Dr Alan Wakeling (Astra-Zeneca Pharmaceuticals, Alderly Park, Cheshire, UK), or vehicle (ethanol) for $24 \mathrm{~h}$. Cells were harvested and S phase was analysed by propidium iodide staining and flow cytometry.

\section{Statistical analyses}

Statistical analyses were performed using Statview 5.0. Software (Abacus Systems, Berkeley, CA, USA). A $P$-value $<0.05$ was accepted as statistically significant. BAG-1 mRNA and protein expression and its association with clinicopathological variables and intrinsic molecular phenotype of breast cancer were tested by applying the $\chi^{2}$-test of association in contingency tables. KaplanMeier and Cox proportional hazards model were used for univariate analysis and the latter for multivariate analyses. Those factors that were prognostic in univariate analysis were then assessed in a multivariable model to identify factors that were independently prognostic and those that were the result of confounding variables.

\section{RESULTS}

\section{BAG-1 mRNA expression and outcome}

To identify an association between BAG-1 gene expression levels and patient outcome, we examined two published breast cancer gene expression data sets. A frequency distribution of BAG-1 mRNA expression was used to apply serial cut points using sequential Kaplan-Meier analysis (log-rank test) to minimise the $P$-value and maximise the difference in survival between the two groups of high and low expressions. Using this approach, statistical significance was assessed for death using univariate Kaplan-Meier and Cox proportional hazards analysis (Table 1). The Wound/NKI data set of 295 patients contained a high BAG-1 expression group of 234 patients (79.3\%), which was associated with improved prognosis in Cox and Kaplan-Meier univariate analysis $(P=0.0005$, Table $1 \mathrm{~A}$ and Figure $1 \mathrm{~A})$. High BAG-1 expression was not significant in a multivariate model that incorporated standard clinicopathological variables (Table 1B).

Table I Association between BAG-I mRNA expression and breast cancer outcome

\begin{tabular}{|c|c|c|c|c|}
\hline \multicolumn{5}{|c|}{$\begin{array}{l}\text { (A) Cox univariate analysis for high BAG-I expression from publicly available gene expression data sets } \\
\text { Wound/NKI }\end{array}$} \\
\hline (Naderi et al, 2007) & 108/135 (80\%) & 0.412 & $0.212-0.843$ & 0.0151 \\
\hline \multicolumn{5}{|c|}{ (B) Cox multivariate analysis for the Wound cohort $(n=295)$} \\
\hline ER positive & & 0.549 & $0.323-0.933$ & 0.0267 \\
\hline HER-2 positive & & 2.319 & $1.267-4.244$ & 0.0064 \\
\hline BAG-I high & & 0.911 & $0.530-1.567$ & 0.7363 \\
\hline
\end{tabular}

$\mathrm{Cl}=$ confidence interval; $\mathrm{ER}=$ oestrogen receptor; $\mathrm{HR}=$ hazards ratio. 

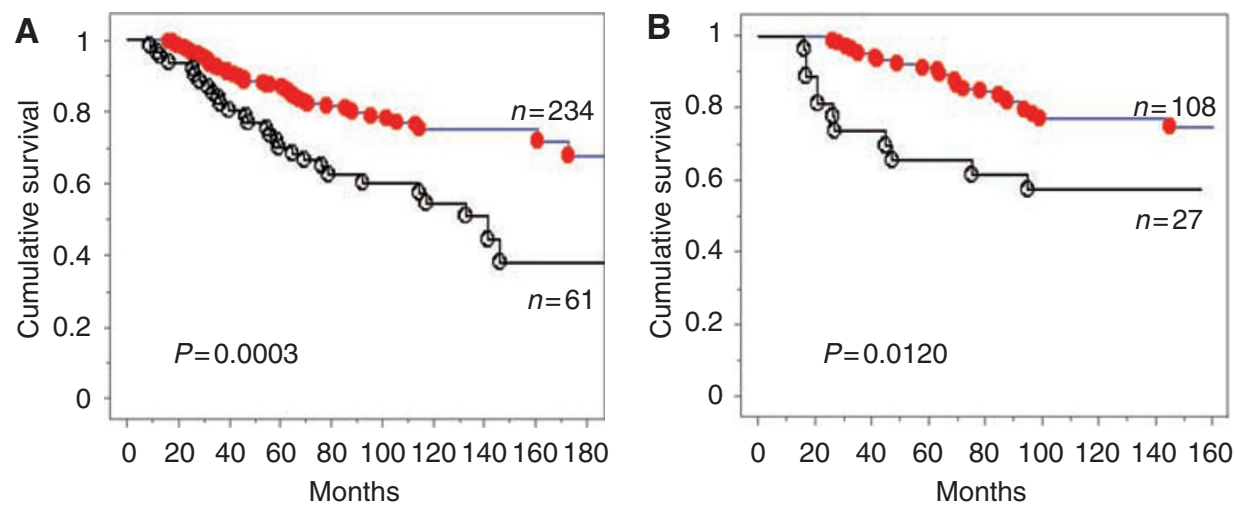

Figure I Relationship between BAG-I mRNA expression and patient outcome. Kaplan-Meier analysis (log-rank test) for breast cancer-specific death in the Wound/NKI (A) and Naderi (B) cohorts. High BAG-I (O); low BAG-I (O).

The Naderi cohort of 135 patients contained a high expression group of 108 patients $(80 \%)$, again associated with a favourable outcome in Kaplan-Meier $(P=0.0120)$ and Cox univariate analyses $(P=0.0151$, Table $1 \mathrm{~A}$ and Figure 1B) but not in multivariate analysis (data not shown). Using these cut points to define high and low expression, further analyses were conducted to determine association between high BAG-1 expression and clinicopathological parameters. In the Wound cohort, high BAG-1 was associated with $\mathrm{ER}+, \mathrm{PR}+$, low histological grade and HER-2 negativity $(P<0.0001)$ but there was no association with tumour size $(P=0.0862)$ or lymph node status $(P>0.999)$. Similarly, the Naderi cohort also showed positive associations between high BAG-1 expression and ER $+(P=0.0014)$, HER-2 negativity $(P=0.0044)$ and low histological grade $(P=0.0061)$ but not with tumour size or lymph node status $(P=0.081$ and $P=0.106$, respectively). These findings support an association of high BAG-1 expression with a luminal A phenotype and improved survival.

\section{Immunohistochemical analysis of BAG-1 protein expression in normal breast tissue and invasive ductal carcinoma}

Representative immunohistochemistry staining patterns and intensities of BAG-1 are illustrated in Figure 2A-I. Similar patterns of staining were observed in normal terminal duct lobular units adjacent to cancer ( $n=24,20$ patients) and in reduction mammoplasty specimens $(n=20,14$ patients). Nuclear staining was observed in all cases, with a mean of $54 \%$ of epithelial cells (range 10-90\%) showing weak-to-strong (1-3+) intensity. Cytoplasmic staining was also present in $63 \%$ of cases with a range of $0-100 \%$ of cells showing 1 or $2+$ staining.

In our cohort of 292 patients, 276 invasive ductal carcinomas were available for BAG-1 analysis due to loss of some tissue cores during processing of the TMAs. Staining was of variable intensity, which ranged from negative to strong $(0-3+)$ and demonstrated both cytoplasmic and nuclear staining in keeping with the known subcellular localisation of the various BAG-1 isoforms (Cutress et al, 2002). There was, however, no direct correlation between nuclear and cytoplasmic expression when modelled as continuous variables $(R=0.476)$. Sixteen out of 276 cases $(5.7 \%)$ showed no nuclear staining, whereas 26 cases $(9.4 \%)$ showed no cytoplasmic staining. When assessed for the percentage of positively staining nuclei, there appeared to be two distinct sub-populations, which could be dichotomised at a cutoff value of $40 \%$ positively staining nuclei at any intensity (Figure 2J). Cytoplasmic staining displayed a similar pattern (Figure $2 \mathrm{~K}$ ), with most tumours showing at least weak positivity but again with two distinct populations that could be identified using a $40 \%$ cutoff value. By applying the selected cut point of $>40 \%$ mean nuclear staining, we defined $78 \%$ (214 out of
276) of the cohort as 'high' BAG-1 expressers and 22\% (62 out of 276) as 'low' BAG-1 expressers. This cut point appeared to represent a real split in the protein expression data, which matched that observed from our analysis of the mRNA expression levels. This distribution was not apparent in the frequency distribution of nuclear ' $H$ ' scores. Consequently, we adopted the percentage of positively staining nuclei as the index for further analysis of association with outcomes.

\section{Correlation of BAG-1 expression with clinicopathological features and intrinsic molecular subtype}

The relationship between nuclear and cytoplasmic expression of BAG-1 and standard clinicopathological features of the disease are summarised in Table 2A. High expression of BAG-1 showed a significant positive correlation with low histological grade, ER and PR positivity $(P<0.0001)$ and was correlated negatively with HER-2 amplification status $(P=0.001)$ and the triple-negative phenotype $(P<0.0001)$. These findings were apparent for both nuclear and cytoplasmic staining at a cut point of $40 \%$ positivity of any intensity, but with a higher degree of statistical significance for nuclear staining. High nuclear BAG-1 expression was also strongly correlated with a luminal A intrinsic phenotype: $73 \%$ (154 out of 211) of BAG-1 'high' were luminal A $\left(P<0.0001, \chi^{2}\right.$-test $)$, but there was no correlation with luminal $\mathrm{B}(P=0.956)$. A strong negative correlation was observed with the HER-2 (5\%, 11 out of 213 BAG-1 high are of HER-2 phenotype, $P<0.0001)$ and the basal-like phenotype $(7 \%, 14$ out of 213 BAG-1 high are basal-like, $P<0.0001$ ).

\section{BAG-1 expression and outcome}

In the whole cohort $(n=276)$, high nuclear BAG-1 expression was associated with a favourable prognosis for all measures of outcome in univariate analysis: local recurrence $(P=0.002)$, distant metastases $(P<0.0001)$ and breast cancer-specific death $(P<0.0001$, Table $3 \mathrm{~A}$ and Figure 3$)$. Furthermore, high nuclear BAG-1 expression was also an independent predictor of outcome in multivariate analysis for distant metastases $(P=0.0455$, Table 3B) but not for local recurrence or death. To assess whether BAG-1 was an independent prognostic variable and not the result of confounding by other variables, Cox proportional hazards models were constructed with step-wise removal of redundant variables until resolution. The resolved multivariate model is presented in Table 3C. High cytoplasmic expression of BAG-1 was also associated with improved outcome for local recurrence $(P=0.0092)$, distant metastases $(P=0.0013)$ and death $(P=0.0046)$ on Kaplan-Meier univariate analysis, but was not significant in multivariate analysis. 

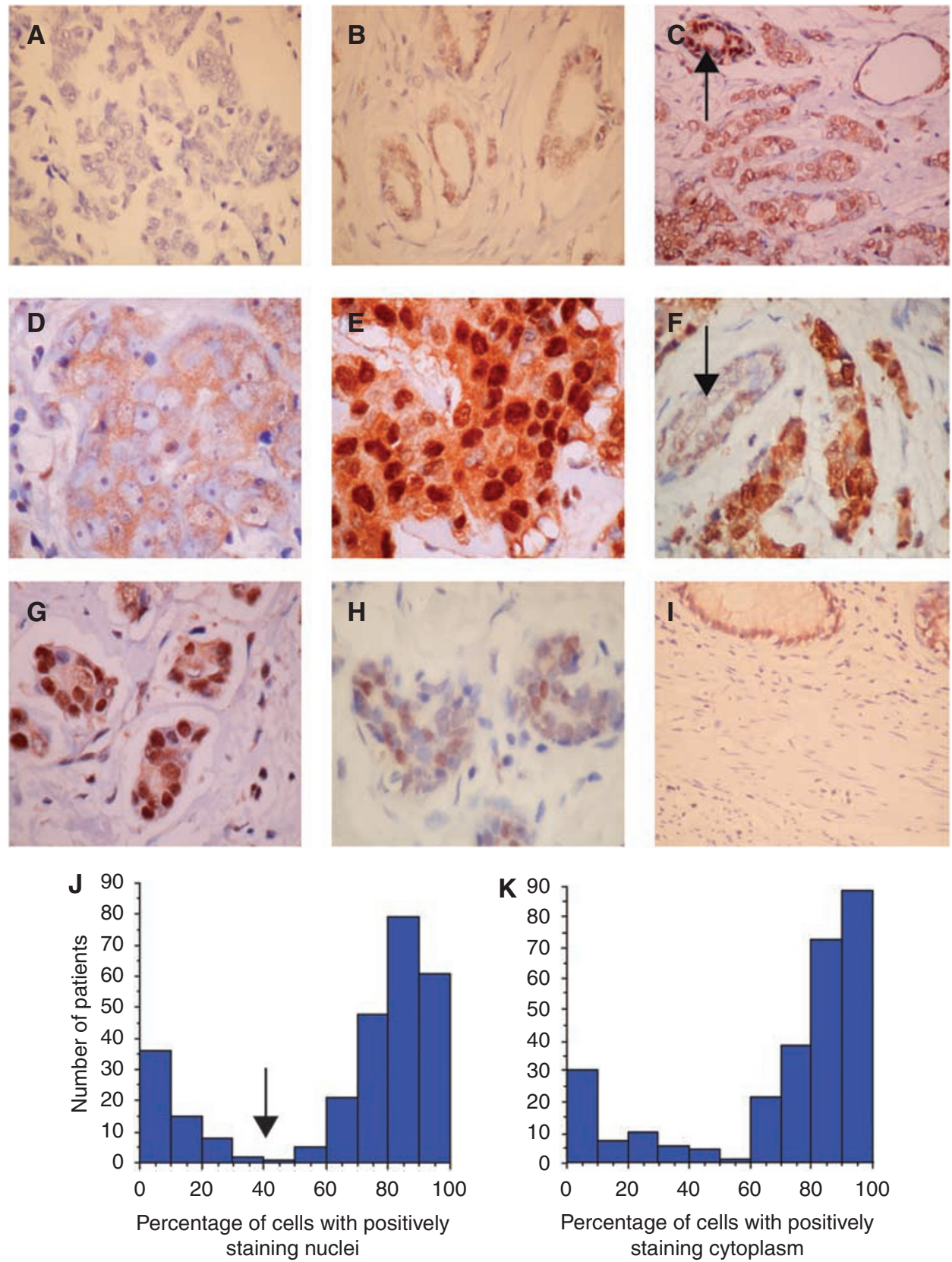

Figure 2 Representative images of BAG-I immunohistochemistry. (A) Negative staining in high-grade invasive ductal carcinoma (IDC), $\times 400$. (B) Weak $(I+)$ nuclear staining in low-grade IDC, $\times 400 .(\mathbf{C})$ Moderate $(2+)$ nuclear and weak $(I+)$ cytoplasmic staining in low-grade IDC, with strong nuclear staining in an adjacent normal duct (arrow). (D) Moderate $(2+)$ cytoplasmic and negative nuclear staining in high-grade IDC. (E) Strong $(3+)$ nuclear and moderate $(2+)$ cytoplasmic staining in high-grade IDC. (F) Strong $(3+)$ nuclear and weak $(1+)$ cytoplasmic staining, weak nuclear staining in normal duct (arrow), $\times 400$. (G) Strong $3+$ nuclear staining. $(\mathbf{H})$ Moderate nuclear staining in normal acini. (I) Normal colon, control tissue, which shows moderate positive nuclear staining in basal crypt cell nuclei and negative staining in mucularis mucosae. Frequency distribution of BAG-I nuclear (J) and cytoplasmic (K) staining using immunohistochemistry in 276 invasive ductal carcinomas. There are two distinct populations that can be dichotomised using a cut point of $40 \%$ (arrow), which segregates the cohort into high- and low-expressing subgroups.

Given the relationship between BAG-1 expression and ER status, we next assessed the association with outcome in the ER + and ER- subgroups. Within ER-positive tumours $(n=189)$, high nuclear BAG-1 expression was an independent predictor of outcome in both univariate and multivariate analyses (Figure 3 and Table 3D). In the multivariate model employed, which incorporated standard pathological indicators of outcome: tumour size, grade, nodal status, PR and HER-2, the resolved model, which eliminates redundant variables, retained HER-2, PR and BAG-1 (Table 3E). Cytoplasmic staining was not significant in univariate analysis in this group of patients. In the smaller subgroup of
ER-negative tumours $(n=85)$, nuclear staining of BAG-1 was not associated with any index of outcome in univariate analysis. As our data demonstrated a strong relationship between high BAG-1 expression, ER positivity and the luminal A phenotype, we assessed whether BAG-1 expression was associated with a differential response to adjuvant tamoxifen therapy. The data reported in Figure 3 demonstrate that patients treated with tamoxifen $(n=107)$, whose tumours had a high nuclear BAG-1 expression, showed an improved outcome in univariate KaplanMeier analysis for local recurrence $(P=0.032)$, distant metastases $(P=0.019)$ and breast cancer-specific death $(P=0.038)$. 
Table 2 Clinicopathological features of the breast cancer cohort and association with BAG-I expression

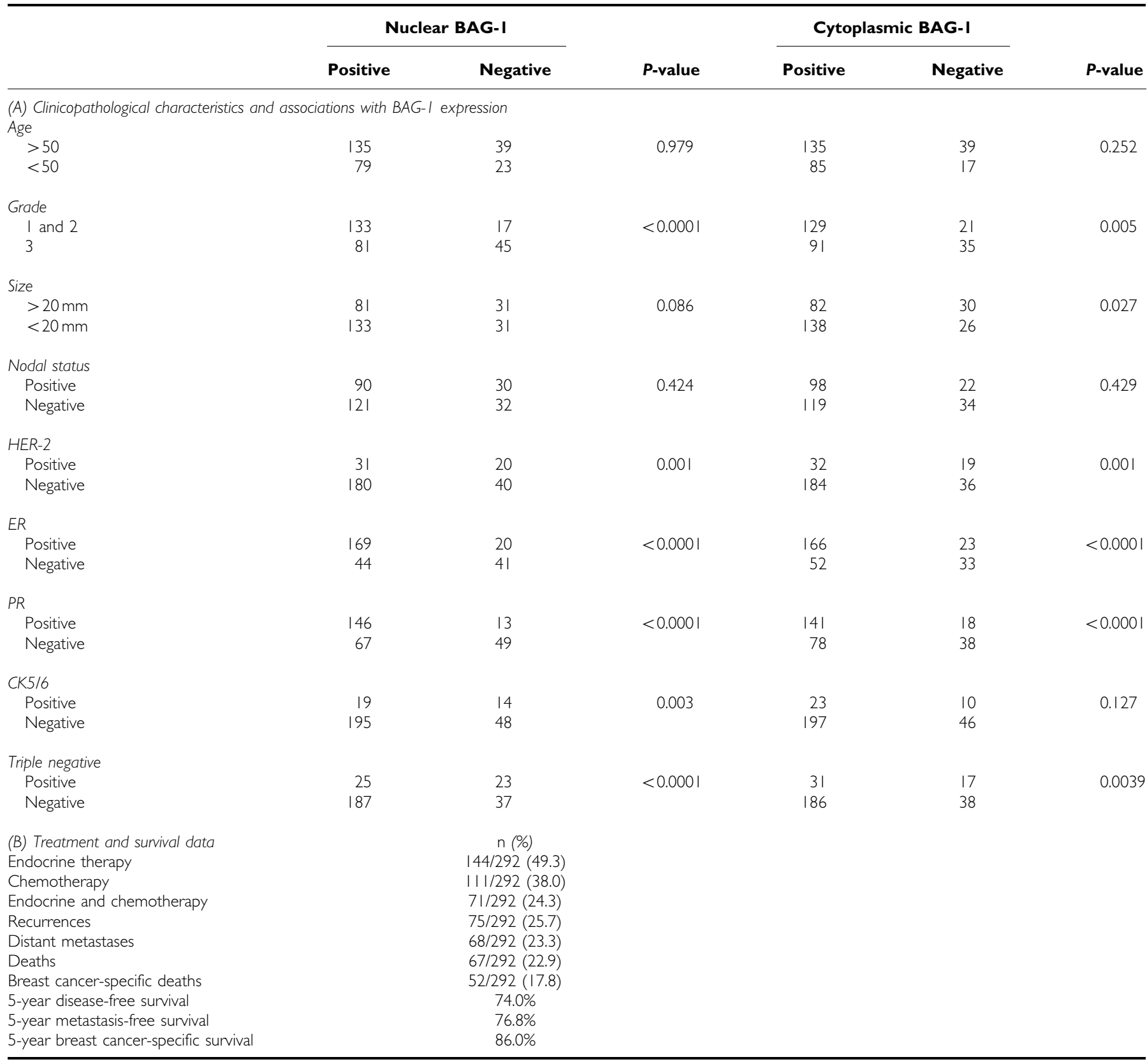

\section{BAG-1 overexpression and antioestrogen sensitivity in vitro}

To provide some potential mechanistic insights into the relationship between high BAG-1 expression and improved outcome in $\mathrm{ER}+$ tamoxifen-treated patients, we assessed the effect of BAG-1 overexpression on oestrogen/antioestrogen sensitivity in ER+ MCF-7 breast cancer cells. A pool of high BAG-1-expressing MCF7 cells was isolated and overexpression of the three major protein isoforms (BAG-1L, BAG-1M and BAG-1S) was confirmed by western blotting (Figure 4A). BAG-1 was also overexpressed in a panel of ER + breast cancer cell lines compared with normal and immortalised breast epithelial cells (Figure 4A), and thus this highexpressing pool of MCF-7 cells represented an appropriate model to study the biological consequences of BAG-1 overexpression and was used for all further analyses.
MCF-7 BAG-1 cells were treated with $1 \mu \mathrm{moll}^{-1}$ 4-hydroxytamoxifen, $10 \mathrm{nmoll}^{-1}$ ICI 182780 or vehicle for $24 \mathrm{~h}$ and the percentage of S-phase cells determined by flow cytometry. Antioestrogen-induced cell cycle arrest was enhanced in MCF-7 BAG-1 cells compared with vector control (Figure 4B). Treatment of MCF-7 BAG-1 cells with the pure oestrogen antagonist ICI $182780(P<0.005)$ or the active metabolite of tamoxifen, 4-hydroxytamoxifen $(P<0.05)$, in replicate experiments demonstrated a significantly enhanced cell cycle arrest as measured by a decrease in $\mathrm{S}$ phase compared with control cells (Figure 4C).

\section{DISCUSSION}

The recent characterisation of molecular phenotypes of breast cancer defines biological subgroups, independent of histological 
Table 3 Cox univariate and multivariate analyses

\begin{tabular}{|c|c|c|c|c|}
\hline & n (\%) & HR & $95 \% \mathrm{Cl}$ & P-value \\
\hline \multicolumn{5}{|c|}{ (A) Whole-cohort clinicopathological variables $(n=292)$, univariate analysis of breast cancer-specific death } \\
\hline Age $>50$ & $184 / 292(63)$ & 1.427 & $0.799-2.551$ & 0.229 \\
\hline Grade $>2$ & $|32 / 29|(45)$ & 3.100 & $1.865-5.163$ & $<0.0001$ \\
\hline Size $>20 \mathrm{~mm}$ & $|17 / 29|(40)$ & 2.730 & $1.678-4.443$ & $<0.0001$ \\
\hline LN positive & $125 / 289(43)$ & 3.968 & $2.346-6.774$ & $<0.0001$ \\
\hline HER-2 positive & $51 / 273(18)$ & 2.459 & $1.463-4.134$ & 0.0007 \\
\hline ER positive & $192 / 280(68)$ & 0.395 & $0.243-0.642$ & 0.0002 \\
\hline PR positive & $|6| / 282(57)$ & 0.238 & $0.140-0.406$ & $<0.0001$ \\
\hline BAG-I high & $214 / 276(78)$ & 0.364 & $0.222-0.598$ & $<0.0001$ \\
\hline \multicolumn{5}{|c|}{ (B) Whole-cohort $(\mathrm{n}=276)$ distant metastases, Cox multivariate analysis } \\
\hline Grade $>2$ & & 1.398 & $0.751-2.567$ & 0.2948 \\
\hline Size $>20 \mathrm{~mm}$ & & 1.564 & $0.937-2.610$ & 0.0873 \\
\hline LN status & & 3.372 & $1.934-5.880$ & $<0.0001$ \\
\hline HER-2 & & 1.853 & $1.066-3.220$ & 0.0287 \\
\hline ER & & 0.990 & $0.525-1.868$ & 0.9745 \\
\hline PR & & 0.405 & $0.212-0.776$ & 0.0064 \\
\hline BAG-I high & & 0.559 & $0.317-0.989$ & 0.0455 \\
\hline \multicolumn{5}{|c|}{ (C) Whole-cohort distant metastases, Cox multivariate analysis, resolved model } \\
\hline LN status & & 3.597 & $2.097-6.168$ & $<0.0001$ \\
\hline HER-2 & & 1.973 & $|| 58-3.36 \mid$. & 0.0125 \\
\hline PR & & 0.329 & $0.186-0.584$ & 0.0001 \\
\hline BAG-I high & & 0.586 & $0.344-0.998$ & 0.0493 \\
\hline \multicolumn{5}{|c|}{ (D) $E R+(\mathrm{n}=189)$ breast cancer-specific death, Cox multivariate analysis } \\
\hline Grade $>2$ & & 1.529 & $0.600-3.896$ & 0.3730 \\
\hline Size $>20 \mathrm{~mm}$ & & 1.053 & $0.428-2.591$ & 0.9100 \\
\hline LN status & & $1.47 \mid$ & $0.57 \mid-3.795$ & 0.4250 \\
\hline HER-2 & & 5.578 & $2.036-15.286$ & 0.0008 \\
\hline PR & & 0.293 & $0.119-0.721$ & 0.0076 \\
\hline BAG-I high & & 0.290 & $0.114-0.735$ & 0.0090 \\
\hline \multicolumn{5}{|c|}{ (E) ER+ breast cancer-specific death, Cox multivariate analysis, resolved model } \\
\hline HER-2 & & 6.725 & $2.7-16.644$ & $<0.0001$ \\
\hline PR & & 0.239 & $0.104-0.547$ & 0.0007 \\
\hline BAG-I high & & 0.302 & $0.122-0.744$ & 0.0093 \\
\hline
\end{tabular}

$\mathrm{Cl}=$ confidence interval; $\mathrm{ER}=$ oestrogen receptor; $\mathrm{HR}=$ hazards ratio.

type, which provides a further insight into the disease at a functional level. Luminal A cancers defined by the presence of ER and/or PR positivity and HER-2 negativity form a favourable prognostic group. However, further defining this group may provide new insights into the underlying biology of oestrogen sensitivity/resistance and provide clinically useful markers for routine clinical practice. This study demonstrates that a high BAG-1 expression identifies a good prognosis group of cancers with a luminal A phenotype, which may have enhanced therapeutic sensitivity to tamoxifen.

We first addressed the question of identifying an association between BAG-1 mRNA expression levels and patient outcome in two independent cohorts, which are broadly equivalent to our validation cohort in terms of clinicopathological characteristics. Using serially determined cut points, we identified two populations of patients with high and low BAG-1 expressions, which correlated with patient outcome. Thus, the high BAG-1 mRNA expression, found within the top $80 \%$ of patients, is associated with a favourable outcome. Correspondingly, the frequency distribution of immunohistochemically detected BAG-1 protein expression in our clinical cohort identifies two distinct subgroups of patients of similar proportions to those identified in the gene expression profiling analyses. High BAG-1 protein expression, defined as greater than $40 \%$ positive nuclear staining of any intensity, identified $78 \%$ of patients with a good prognosis. The predictive value of high BAG-1 expression was greatest in $\mathrm{ER}+$ cancer in which a high nuclear expression was an independent predictor of prognosis for local recurrence, distant metastases and death. Furthermore, for breast cancer-specific death, BAG-1 expression was of superior predictive power to tumour grade, tumour size and lymph node status. This group of patients has a strong positive correlation with a luminal A phenotype and low histological grade, which suggests that BAG-1 may be a useful surrogate marker of intact ER signalling and identifies those tumours maintaining a luminal A-differentiated phenotype. Therefore, BAG-1 is a marker with potentially useful prognostic applications in ER + disease.

Outcome studies, published earlier, of BAG-1 expression using immunohistochemistry have shown inconsistent results but with a trend towards improved prognosis with high expression levels. However, its role as a predictive biomarker has not yet been fully defined or adequately validated. The first published study (Tang et al, 1999) of 140 patients included both early and metastatic disease, ER status was unknown in 38\% of patients and only $35 \%$ of patients were ER + . Consequently, ER and PR were excluded from multivariate analysis, which showed that an elevated nuclear BAG-1 expression was associated with shorter disease-free and overall survival, although BAG-1 was not significant in univariate analysis. These findings were not replicated in a subsequent study by the same group of investigators (Tang et al, 2004). The second study of 122 patients (Turner et al, 2001) consisted predominantly of pre-menopausal patients (mean age, 54 years), only $41 \%$ of whom had ER + cancers, and lymph node status was unknown in $48 \%$ of cases. In addition, well-documented prognostic indicators, such as tumour size, grade, ER, PR and HER-2, were not significant 

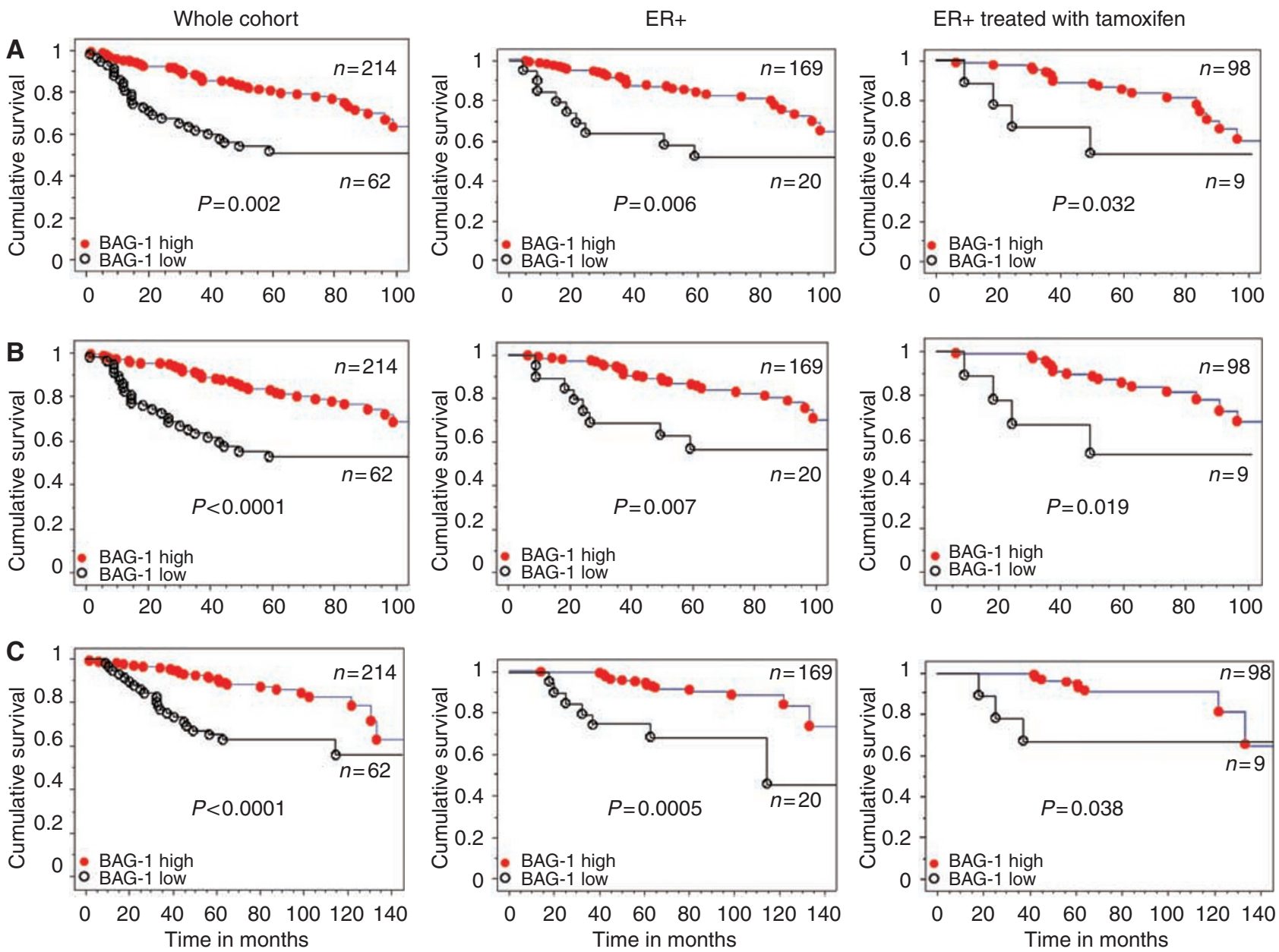

Figure 3 Relationship between nuclear BAG-I protein expression by immunohistochemistry and patient outcome. Kaplan-Meier analyses (log-rank test) for $(\mathbf{A})$ local recurrence, $(\mathbf{B})$ distant metastases and $(\mathbf{C})$ breast cancer-specific death in the whole cohort, ER + subgroup and ER + patients treated with tamoxifen stratified by high $(\mathbf{O})$ and low $(\bigcirc)$ BAG-I expression.

in univariate analysis. In this study, elevated cytoplasmic BAG-1 expression was associated with improved prognosis in a multivariate model that included $\mathrm{ER}, \mathrm{BCL}-2$ and stage. In a more homogeneous and representative cohort of early breast cancer, Cutress et al (2003) described improved prognosis in univariate analysis with high nuclear, but not high cytoplasmic, BAG-1 expression in a cohort of 138 patients, $60 \%$ of whom were ER + . All patients were treated with surgery and endocrine therapy without chemotherapy. The largest and the most recent study of 517 patients (Nadler et al, 2008) used image analysis-based assessment of immunofluorescent staining and found that both high nuclear and cytoplasmic expression of BAG-1 was associated with improved prognosis in the whole cohort and in lymph nodepositive patients only in univariate analysis, with a strong correlation with ER, PR and Bcl-2. However, again in this study, only $52 \%$ of patients were $\mathrm{ER}+$, with a predominance of large tumours $(59 \%>2 \mathrm{~cm})$, and details on histological grade and treatment were not available. Two other studies were unable to identify any association with outcome in patients treated with hormonal therapy (Townsend et al, 2002) or in a cohort with advanced breast cancer treated with chemotherapy (Sjostrom et al, 2002). There are many possible explanations for these discordant findings: differences in the composition of the clinical cohorts, incomplete clinical information, different antigen retrieval methods, differing monoclonal antibodies used in the detection of BAG-1 and divergent cut points used to determine a high or low expression. This study, therefore, confirms the findings described earlier of improved prognosis with high nuclear BAG-1 expression described by Cutress et al (2003) and represents a detailed analysis of BAG-1 expression and its potential relationship with therapeutic responsiveness in a large cohort of uniform histological type with well-documented clinical outcome.

The finding of improved responsiveness to tamoxifen and better patient outcome associated with a high expression of BAG-1, a pro-survival antiapoptotic protein, is somewhat counter-intuitive but is mirrored by several studies identifying the overexpression of BCL-2, a major target of BAG-1, also being consistently associated with improved prognosis in low-grade ER + tumours (Callagy et al, 2006) and also in patients treated with tamoxifen (Linke et al, 2006). Furthermore, the strong relationship between high nuclear BAG-1 expression and improved patient outcome reported by Cutress et al (2003) emanated from a cohort of tamoxifen-treated patients. $B A G-1, B C L-2$ and $E R$ feature among the 16 cancerrelated genes of the Oncotype Dx assay (Paik et al, 2004), which predicts distant failure in ER + lymph node-negative patients treated with tamoxifen. The derived recurrence-score algorithm, which is largely weighted towards proliferation-related genes, assigns a negative value to the BAG-1 mRNA expression level, in turn supporting our observation of improved prognosis with high expression level. Several other gene expression profiling studies have identified signatures predictive of outcome in ER + disease treated with tamoxifen (Ma et al, 2004; Jansen et al, 2005; Loi et al, 
A
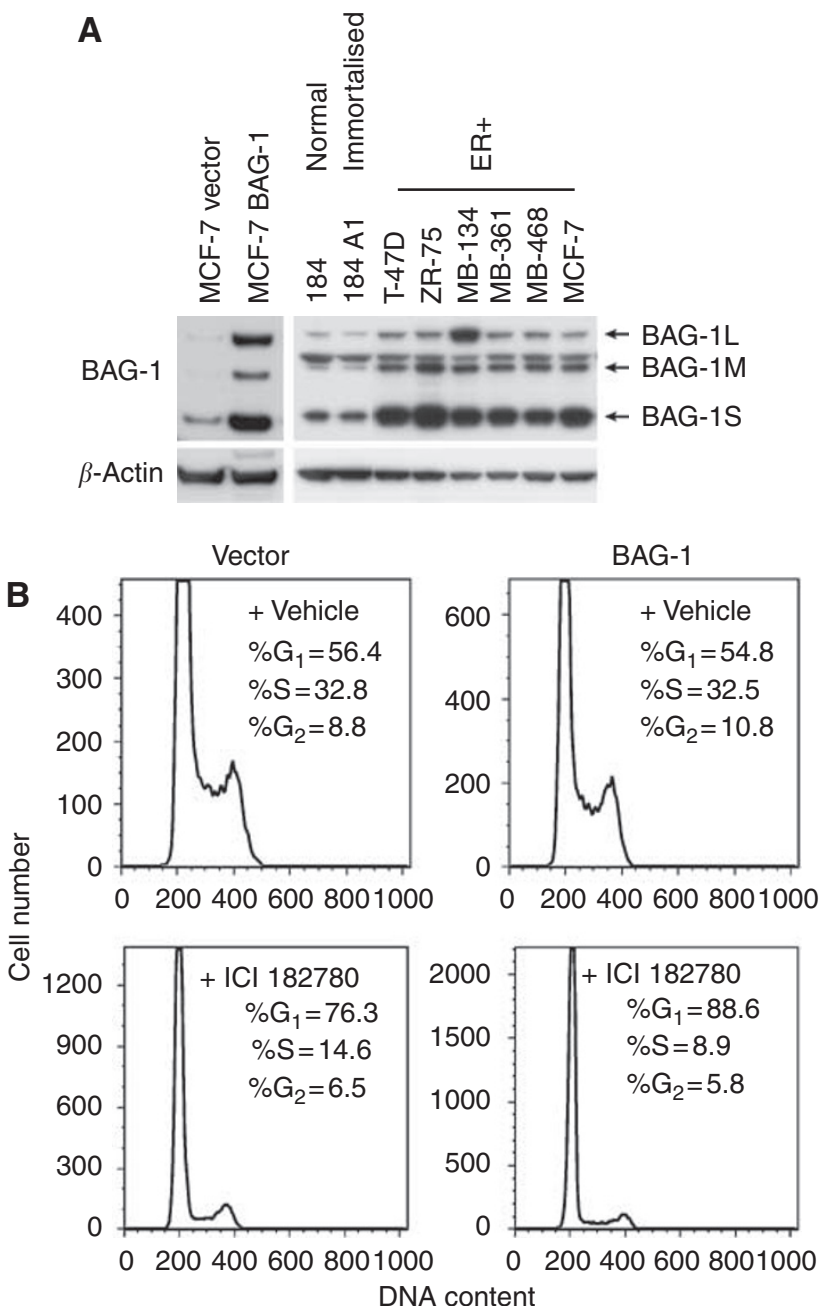

DNA content

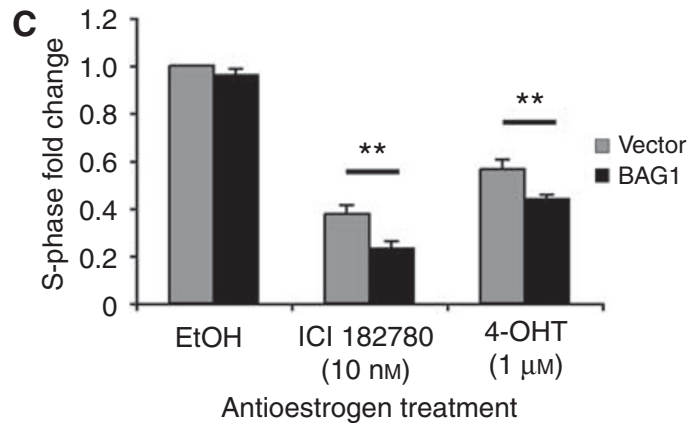

Figure 4 Effects of modulating BAG-I expression on antioestrogen sensitivity in MCF-7 breast cancer cells. (A) Immunoblot analysis of cell lysates from MCF-7 retrovirally infected pools stably overexpressing BAG-I wild type, two normal breast epithelial and six ER + breast cancer cell lines. $\beta$-Actin was used as a loading control. Each of the three major BAG-I protein isoforms are indicated: BAG-IL, BAG-IM and BAG-IS. (B) Representative DNA histograms of MCF-7 cells stably overexpressing BAG-I compared with control cells after treatment with $\left.10 \mathrm{nmol}\right|^{-1} \mid \mathrm{Cl}$ I 82780 or vehicle for $24 \mathrm{~h}$. Differences in scale are due to slight differences in the number of events recorded. (C) Proliferating MCF-7 cells stably overexpressing BAG-I were treated with $\mid \mu$ mol I $^{-1}$ 4-hydroxytamoxifen, $10 \mathrm{nmol}^{-1} \mathrm{ICl} \mid 82780$ or vehicle for $24 \mathrm{~h}$. Cells were harvested and $\mathrm{S}$ phase was analysed by propidium iodide staining and flow cytometry. The decrease in $\mathrm{S}$ phase was graphed as fold change relative to vehicle-treated vector control cells. The bar histograms represent the mean \pm s.e.m. for replicate samples from five independent experiments. $* P<0.05$; *** $P<0.005$.
2008). BAG-1 did not feature among genes within these signatures, although there is often a limited overlap in signatures between studies (Miller, 2007). Interestingly, an expression profiling study (Cleator et al, 2006) of pre-treatment biopsies from 40 patients treated with AC chemotherapy identified $B A G-1, B C L-2$ and $E R$ among a diverse group of 178 genes overexpressed in sensitive tumours. Thus, the potential role of BAG-1 as a predictive marker of therapeutic responsiveness to both endocrine and chemotherapy requires further investigation. This is best performed within the context of randomised clinical trials and these studies are ongoing.

The BAG-1 gene is located on chromosome 9p12 and is expressed as three protein isoforms generated through alternative initiation sites from a single mRNA (Packham et al, 1997). The overexpression of BAG-1 has been described in several breast cancer cell lines (Takayama et al, 1998; Brimmell et al, 1999), with the three isoforms demonstrating differing intracellular localisations: BAG-1L is predominantly nuclear, BAG-1S is predominantly cytoplasmic and BAG-1M is present in both the cellular compartments (Brimmell et al, 1999). This differential subcellular localisation of BAG-1 isoforms is altered under different experimental conditions, possibly representing a regulatory mechanism for protein activity: BAG-1M relocalises from the cytoplasm to the nucleus following heat shock (Zeiner et al, 1999) and when bound to the glucocorticoid receptor, possibly downregulating the receptor (Schneikert et al, 1999). Nuclear-to-cytoplasmic relocalisation of BAG-1M has also been observed during epidermal and neuronal differentiation and during breast epithelial involution, both in vitro and in vivo (Takayama et al, 1998; Schorr et al, 1999; Kermer et al, 2002). BAG-1 possesses a range of pro-survival properties through its ability to interact with diverse downstream target molecules, originally described by its ability to bind to and enhance the activity of the antiapoptotic protein BCL-2 mediated by its binding to the heat-shock proteins HSP70 and HSC70 (Takayama et al, 1995). Differential staining and subcellular localisation of the isoforms have not been investigated in the normal breast or breast cancer and are impaired by the absence of isoform-specific antibodies, all current studies in breast detecting 'total' BAG-1 expression. The mechanism of overexpression of BAG-1 in breast cancer is also not known although in prostate cancer it is amplified in $7.4 \%$ of hormone-refractory cancers (Maki et al, 2007). Our data with normal and malignant breast epithelial cell lines confirm overexpression in ER + carcinoma cell lines compared with normal epithelial cells. The BAG-1S isoform appeared to be preferentially overexpressed, but there was evidence for elevated expression of all three isoforms in a cell line-specific manner. The relative contribution of the respective isoforms to the relationships reported here must await further studies with isoform-specific antibodies.

A key target of BAG-1 is ER $\alpha$, which when bound by the BAG-1L isoform increases its transcriptional activity by up to five-fold in MCF-7 cells (Cutress et al, 2003). The ability of high nuclear BAG-1 expression to predict improved outcome in ER + cancer and also in those treated with tamoxifen is of potential mechanistic importance as it suggests that it may have a role in responsiveness to adjuvant endocrine therapy. In our subgroup of ER + patients treated with tamoxifen $(n=107)$, high BAG-1 expression predicted improved prognosis, which may indicate sensitivity to therapy or possibly a better definition of a good prognostic luminal A group of patients. To address the question of whether BAG-1 expression could confer enhanced sensitivity to antioestrogen treatment in vitro, we analysed cell cycle arrest in BAG-1-overexpressing MCF-7 cells. The data presented here demonstrate a significant increase in sensitivity to the induction of cell cycle arrest by both tamoxifen and the pure steroidal antioestrogen ICI 182780.

As antioestrogen therapy, targeted at oestrogen synthesis (aromatase inhibitors), or the ER (tamoxifen), is the single mosteffective treatment for women with hormone receptor-positive 
disease, the ability to predict likely success or failure of these therapies would enable potential alternative therapeutic strategies to be targeted to a group of patients most likely to fail on tamoxifen or aromatase therapy up-front or at an earlier stage in treatment, which may result in improved outcome. The ability of BAG-1 to predict responsiveness to antioestrogen therapy now merits further investigation by examining the relationship between expression and response in large randomised clinical trials of endocrine therapy in ER + patients.

In summary, we have demonstrated that the high BAG-1 expression is associated with the luminal A phenotype, is an independent predictor of outcome and may indicate enhanced responsiveness to tamoxifen in $\mathrm{ER}+$ invasive ductal carcinoma. These effects may be related to the ability of BAG-1 overexpression to confer increased sensitivity to antioestrogens in vitro. These findings suggest that BAG-1 immunohistochemistry may have a role in a routine pathology setting as a marker for better defining luminal A breast cancers and as a therapeutic response marker for ER-targeted therapy with tamoxifen or aromatase inhibitors.

\section{ACKNOWLEDGEMENTS}

This study was supported by grants from the Cancer Institute NSW, the National Health and Medical Research Council of Australia (NHMRC), the Petre Foundation, the RT Hall Trust. We thank Ms Joanne Scorer for assistance in preparing the paper and Drs Davendra Segara, Dianne Adams, Andrew Field and Ms Alice Boulghourjian for assistance with the collection and processing of the tissue, the establishment of the breast cancer database and data management.

\section{REFERENCES}

Brimmell M, Burns JS, Munson P, McDonald L, O'Hare MJ, Lakhani SR, Packham G (1999) High level expression of differentially localized BAG-1 isoforms in some oestrogen receptor-positive human breast cancers. Br J Cancer 81: 1042 - 1051

Caldon CE, Swarbrick A, Lee CS, Sutherland RL, Musgrove EA (2008) The helix-loop-helix protein Id1 requires cyclin D1 to promote the proliferation of mammary epithelial cell acini. Cancer Res 68: 3026-3036

Callagy GM, Pharoah PD, Pinder SE, Hsu FD, Nielsen TO, Ragaz J, Ellis IO, Huntsman D, Caldas C (2006) Bcl-2 is a prognostic marker in breast cancer independently of the Nottingham Prognostic Index. Clin Cancer Res 12: $2468-2475$

Cheang MC, Voduc D, Bajdik C, Leung S, McKinney S, Chia SK, Perou CM, Nielsen TO (2008) Basal-like breast cancer defined by five biomarkers has superior prognostic value than triple-negative phenotype. Clin Cancer Res 14: $1368-1376$

Cleator S, Tsimelzon A, Ashworth A, Dowsett M, Dexter T, Powles T, Hilsenbeck S, Wong H, Osborne CK, O'Connell P, Chang JC (2006) Gene expression patterns for doxorubicin (Adriamycin) and cyclophosphamide (cytoxan) (AC) response and resistance. Breast Cancer Res Treat 95: $229-233$

Cutress RI, Townsend PA, Brimmell M, Bateman AC, Hague A, Packham G (2002) BAG-1 expression and function in human cancer. Br J Cancer 87: $834-839$

Cutress RI, Townsend PA, Sharp A, Maison A, Wood L, Lee R, Brimmell M, Mullee MA, Johnson PW, Royle GT, Bateman AC, Packham G (2003) The nuclear BAG-1 isoform, BAG-1L, enhances oestrogen-dependent transcription. Oncogene 22: 4973-4982

Debnath J, Muthuswamy SK, Brugge JS (2003) Morphogenesis and oncogenesis of MCF-10A mammary epithelial acini grown in threedimensional basement membrane cultures. Methods 30: 256-268

Early Breast Cancer Trialists' Collaborative Group (EBCTCG) (2005) Effects of chemotherapy and hormonal therapy for early breast cancer on recurrence and 15-year survival: an overview of the randomised trials. Lancet 365: 1687-1717

Elston CW, Ellis IO (1991) Pathological prognostic factors in breast cancer. I. The value of histological grade in breast cancer: experience from a large study with long-term follow-up. Histopathology 19: 403-410

Fisher B, Costantino JP, Wickerham DL, Redmond CK, Kavanah M, Cronin WM, Vogel V, Robidoux A, Dimitrov N, Atkins J, Daly M, Wieand S, Tan-Chiu E, Ford L, Wolmark N (1998) Tamoxifen for prevention of breast cancer: report of the National Surgical Adjuvant Breast and Bowel Project P-1 Study. J Natl Cancer Inst 90: 1371 - 1388

Goldhirsch A, Wood WC, Gelber RD, Coates AS, Thurlimann B, Senn HJ (2007) Progress and promise: highlights of the international expert consensus on the primary therapy of early breast cancer 2007. Ann Oncol 18: $1133-1144$

Howell A, Cuzick J, Baum M, Buzdar A, Dowsett M, Forbes JF, Hoctin-Boes G, Houghton J, Locker GY, Tobias JS (2005) Results of the ATAC (Arimidex, Tamoxifen, Alone or in Combination) trial after completion of 5 years' adjuvant treatment for breast cancer. Lancet 365: 60-62

Jansen MP, Foekens JA, van Staveren IL, Dirkzwager-Kiel MM, Ritstier K, Look MP, Meijer-van Gelder ME, Sieuwerts AM, Portengen H, Dorssers
LC, Klijn JG, Berns EM (2005) Molecular classification of tamoxifenresistant breast carcinomas by gene expression profiling. J Clin Oncol 23: $732-740$

Kermer P, Krajewska M, Zapata JM, Takayama S, Mai J, Krajewski S, Reed JC (2002) Bag1 is a regulator and marker of neuronal differentiation. Cell Death Differ 9: $405-413$

Kitada S, Andersen J, Akar S, Zapata JM, Takayama S, Krajewski S, Wang HG, Zhang X, Bullrich F, Croce CM, Rai K, Hines J, Reed JC (1998) Expression of apoptosis-regulating proteins in chronic lymphocytic leukemia: correlations with in vitro and in vivo chemoresponses. Blood 91: $3379-3389$

Linke SP, Bremer TM, Herold CD, Sauter G, Diamond C (2006) A multimarker model to predict outcome in tamoxifen-treated breast cancer patients. Clin Cancer Res 12: 1175-1183

Loi S, Haibe-Kains B, Desmedt C, Wirapati P, Lallemand F, Tutt AM, Gillet C, Ellis P, Ryder K, Reid JF, Daidone MG, Pierotti MA, Berns EM, Jansen MP, Foekens JA, Delorenzi M, Bontempi G, Piccart MJ, Sotiriou C (2008) Predicting prognosis using molecular profiling in estrogen receptor-positive breast cancer treated with tamoxifen. BMC Genomics 9: 239

Ma XJ, Wang Z, Ryan PD, Isakoff SJ, Barmettler A, Fuller A, Muir B, Mohapatra G, Salunga R, Tuggle JT, Tran Y, Tran D, Tassin A, Amon P, Wang W, Wang W, Enright E, Stecker K, Estepa-Sabal E, Smith B, Younger J, Balis U, Michaelson J, Bhan A, Habin K, Baer TM, Brugge J, Haber DA, Erlander MG, Sgroi DC (2004) A two-gene expression ratio predicts clinical outcome in breast cancer patients treated with tamoxifen. Cancer Cell 5: 607-616

Maki HE, Saramaki OR, Shatkina L, Martikainen PM, Tammela TL, van Weerden WM, Vessella RL, Cato AC, Visakorpi T (2007) Overexpression and gene amplification of BAG-1L in hormone-refractory prostate cancer. J Pathol 212: 395-401

Miller WR (2007) Do classical oestrogen markers predict for clinical response to endocrine therapy? Breast Cancer Res 9: S19

Naderi A, Teschendorff AE, Barbosa-Morais NL, Pinder SE, Green AR, Powe DG, Robertson JF, Aparicio S, Ellis IO, Brenton JD, Caldas C (2007) A gene-expression signature to predict survival in breast cancer across independent data sets. Oncogene 26: 1507-1516

Nadler Y, Camp RL, Giltnane JM, Moeder C, Rimm DL, Kluger HM, Kluger $\mathrm{Y}$ (2008) Expression patterns and prognostic value of Bag-1 and Bcl-2 in breast cancer. Breast Cancer Res 10: R35

Packham G, Brimmell M, Cleveland JL (1997) Mammalian cells express two differently localized Bag-1 isoforms generated by alternative translation initiation. Biochem J 328(Part 3): 807-813

Paik S, Shak S, Tang G, Kim C, Baker J, Cronin M, Baehner FL, Walker MG, Watson D, Park T, Hiller W, Fisher ER, Wickerham DL, Bryant J, Wolmark N (2004) A multigene assay to predict recurrence of tamoxifentreated, node-negative breast cancer. $N$ Engl J Med 351: 2817-2826

Paik S, Tang G, Shak S, Kim C, Baker J, Kim W, Cronin M, Baehner FL, Watson D, Bryant J, Costantino JP, Geyer Jr CE, Wickerham DL, Wolmark N (2006) Gene expression and benefit of chemotherapy in women with node-negative, estrogen receptor-positive breast cancer. J Clin Oncol 24: $3726-3734$ 
Prall OW, Sarcevic B, Musgrove EA, Watts CK, Sutherland RL (1997) Estrogen-induced activation of Cdk4 and Cdk2 during G1-S phase progression is accompanied by increased cyclin D1 expression and decreased cyclin-dependent kinase inhibitor association with cyclin E-Cdk2. J Biol Chem 272: $10882-10894$

R Development Core Team (2007) R: A Langauge and Environment for Statistical Computing. In $\mathrm{R}$ Foundation for Statistical Computing: Vienna, Austria

Schneikert J, Hubner S, Martin E, Cato AC (1999) A nuclear action of the eukaryotic cochaperone RAP46 in downregulation of glucocorticoid receptor activity. J Cell Biol 146: 929-940

Schorr K, Li M, Krajewski S, Reed JC, Furth PA (1999) Bcl-2 gene family and related proteins in mammary gland involution and breast cancer. J Mammary Gland Biol Neoplasia 4: 153-164

Shindoh M, Adachi M, Higashino F, Yasuda M, Hida K, Nishioka T, Ono M, Takayama S, Reed JC, Imai K, Totsuka Y, Kohgo T (2000) BAG-1 expression correlates highly with the malignant potential in early lesions (T1 and T2) of oral squamous cell carcinoma. Oral Oncol 36: 444-449

Sjostrom J, Blomqvist C, von Boguslawski K, Bengtsson NO, Mjaaland I, Malmstrom P, Ostenstadt B, Wist E, Valvere V, Takayama S, Reed JC, Saksela E (2002) The predictive value of bcl-2, bax, bcl-xL, bag-1, fas, and fasL for chemotherapy response in advanced breast cancer. Clin Cancer Res 8: $811-816$

Smyth GK (2005) Limma: linear models for microarray data. In Bioinformatics and Computational biology solutions using $R$ and Bioconductor, Gentlemen R, Carey V, Dudoit S, Irizarry R, Huber W (eds), pp 397-420. Springer: New York

Sorlie T, Perou CM, Tibshirani R, Aas T, Geisler S, Johnsen H, Hastie T, Eisen MB, van de Rijn M, Jeffrey SS, Thorsen T, Quist H, Matese JC, Brown PO, Botstein D, Eystein Lonning P, Borresen-Dale AL (2001) Gene expression patterns of breast carcinomas distinguish tumor subclasses with clinical implications. Proc Natl Acad Sci USA 98: 10869-10874
Takayama S, Krajewski S, Krajewska M, Kitada S, Zapata JM, Kochel K, Knee D, Scudiero D, Tudor G, Miller GJ, Miyashita T, Yamada M, Reed JC (1998) Expression and location of Hsp70/Hsc-binding anti-apoptotic protein BAG-1 and its variants in normal tissues and tumor cell lines. Cancer Res 58: 3116-3131

Takayama S, Sato T, Krajewski S, Kochel K, Irie S, Millan JA, Reed JC (1995) Cloning and functional analysis of BAG-1: a novel Bcl-2-binding protein with anti-cell death activity. Cell 80: 279-284

Tang SC, Beck J, Murphy S, Chernenko G, Robb D, Watson P, Khalifa M (2004) BAG-1 expression correlates with Bcl-2, p53, differentiation, estrogen and progesterone receptors in invasive breast carcinoma. Breast Cancer Res Treat 84: 203-213

Tang SC, Shehata N, Chernenko G, Khalifa M, Wang X (1999) Expression of BAG-1 in invasive breast carcinomas. J Clin Oncol 17: 1710 - 1719

Townsend PA, Dublin E, Hart IR, Kao RH, Hanby AM, Cutress RI, Poulsom R, Ryder K, Barnes DM, Packham G (2002) BAG-i expression in human breast cancer: interrelationship between BAG-1 RNA, protein, HSC70 expression and clinico-pathological data. J Pathol 197: $51-59$

Turner BC, Krajewski S, Krajewska M, Takayama S, Gumbs AA, Carter D, Rebbeck TR, Haffty BG, Reed JC (2001) BAG-1: a novel biomarker predicting long-term survival in early-stage breast cancer. J Clin Oncol 19: $992-1000$

van de Vijver MJ, He YD, van't Veer LJ, Dai H, Hart AA, Voskuil DW, Schreiber GJ, Peterse JL, Roberts C, Marton MJ, Parrish M, Atsma D, Witteveen A, Glas A, Delahaye L, van der Velde T, Bartelink H, Rodenhuis S, Rutgers ET, Friend SH, Bernards R (2002) A geneexpression signature as a predictor of survival in breast cancer. $N$ Engl $J$ Med 347: 1999-2009

Zeiner M, Niyaz Y, Gehring U (1999) The hsp70-associating protein Hap46 binds to DNA and stimulates transcription. Proc Natl Acad Sci USA 96: $10194-10199$ 